Recruitment of all 48 patients in the trial will complete in early 2012 and results to date are presented descriptively here.

Methods Patients with impaired quality of life due to active Crohn's disease, despite at least three immunosuppressive agents all receive mobilisation treatment (intravenous cyclophosphamide $4 \mathrm{gm} / \mathrm{m}^{2}$ over 2 days followed by recombinant human granulocyte-colony stimulating factor [GCSF, filgrastim], $10 \mu / \mathrm{kg}$ daily before randomisation to immediate (1 month) or delayed (1 year) immunoablation and stem cell transplantation. The conditioning regime is intravenous cyclophosphamide $50 \mathrm{mg} / \mathrm{kg}$ per day for 4 days, antithymocyte globulin $2.5 \mathrm{mg} / \mathrm{kg} /$ day and methyl prednisolone $1 \mathrm{mg} /$ $\mathrm{kg}$ on days $3-5$. The bone marrow is reconstituted by infusion of an unselected graft of $3-8 \times 106 / \mathrm{kg}$ CD34 positive stem cells. Results are compared 1 year after mobilisation alone or after transplantation.

Results Twelve months after stem cell transplantation (early or delayed) the Crohn's Disease Activity Index (CDAI) fell from 324 (median, IOR 229-411) to $161(85-257, \mathrm{n}=17)$ compared to 351 (287-443) to 272 (214-331) following mobilisation alone $(n=11)$. Six patients had a normal CDAI after transplantation vs one after mobilisation. C reactive protein fell from $16.6(6.7-32.0) \mathrm{mg} / \mathrm{l}$ to 6.5 $(3.5-12.5) \mathrm{mg} / \mathrm{l}$ vs $14(8.0-27.0) \mathrm{mg} / \mathrm{l}$ to 9.0 (2.0-23.4) mg/l following mobilisation alone. The Crohn's Disease Endoscopic Index of Severity (CDEIS) (aggregate for upper and lower endoscopy) fell from $18(10-25)$ to $5(1-11)$ following transplantation vs 14 (12-16) to 9 (4-22) following mobilisation. Three patients achieved the goal of a normal CDAI, no drug therapy and normal upper and lower endoscopy 1 year after tranplantation but so did one patient following mobilisation alone. Serious Adverse Events were common ( $n=100$ to date) with 42 infective episodes requiring or prolonging hospitalisation, following both mobilisation and conditioning and transplantation. There were seven episodes of viral (re)activation. Temporary flare of Crohn's disease activity or a need for surgery occurred in eight patients.

Conclusion Immunoablation and hemopoietic stem cell transplantation appears to be an effective treatment for some patients with Crohn's Disease, although full results will be required for a firm conclusion. Risks are significant, making it potentially suitable for only a limited number of patients. Data from the whole trial will be needed to judge whether mobilisation alone has any benefits.

Competing interests None declared.

\section{BSG endoscopy section free papers OC-044 GASTROSCOPY RATE IN ENGLISH GENERAL PRACTICE POPULATIONS: ASSOCIATION WITH OUTCOME FOR OESOPHAGOGASTRIC CANCER}

doi:10.1136/gutjnl-2012-302514a.44

${ }^{1} \mathrm{M}$ Shawihdi, ${ }^{*}{ }^{1} \mathrm{G}$ Powell, ${ }^{2} \mathrm{~N}$ Stern, ${ }^{2} \mathrm{~N}$ Kapoor, ${ }^{2} \mathrm{R}$ Sturgess, ${ }^{1} \mathrm{E}$ Thompson, ${ }^{1} \mathrm{M}$ Pearson, ${ }^{3} \mathrm{~K}$ Bodger. ${ }^{1}$ Aintree Health Outcomes Partnership, University of Liverpool, Liverpool, UK; ${ }^{2}$ Digestive Diseases Centre, Aintree University Hospital, Liverpool, UK; ${ }^{3}$ Department of Gastroenterology, Institute of Translational Medicine, University of Liverpool, Liverpool, UK

Introduction Rates of gastroscopy vary between English general practice populations. The magnitude of this variation suggests a wide spectrum of clinical practice. Current guidelines focus on alarm symptoms as triggers for investigation but early symptoms of cancer are non-specific. This project aimed to determine whether overall gastroscopy rate in GP practice populations in England is associated with outcome of oesophagogastric cancer (OGC), as measured by rate of major surgical resection, emergency admission for cancer diagnosis and mortality.

Methods Analysis of Hospital Episode Statistics (HES, 2006-2008) linked to death registry and practice population data. Gastroscopy volume determined by extracting total diagnostic gastroscopy procedures and aggregated at GP practice level. OGC cases: Methods developed and validated (using local \& national audit) to identify new cases of OGC and then extract all hospital episodes in chronological order, flag key milestones (eg, diagnostic gastroscopy; emergency admission to hospital; major surgery) using relevant diagnostic and procedure codes. Entry criteria: General practices with $\geq 1$ new case of OGC and with a per capita gastroscopy rate within a valid reference range (0.4-4.0 per 1000 population). Practices grouped into tertiles (low, medium and high gastroscopy rate).

Results 20709 OGC cases from 5956 practices serving an adult population of 35.1 million. Characteristics of OGC cases matched the national audit findings. Cases registered with practices in lowest tertile of gastroscopy rate had lowest rate of surgery (14\% vs $16 \%$ vs $16 \% ; \mathrm{p}=0.028)$, highest rate of emergency admission ( $29 \%$ vs $27 \%$ vs $25 \% ; \mathrm{p}<0.01$ ), and highest mortality at 6 months ( $41 \%$ vs $40 \%$ vs $39 \% ; \mathrm{p}<0.01)$. After adjustment for age, sex, co-morbidity and deprivation quintile in logistic regression analysis, the rate of gastroscopy (low, medium or high) at the patient's general practice was an independent predictor of all three outcomes.

Conclusion There is $>10$-fold variation in the rate of gastroscopy among general practice populations in England. On average, OGC patients belonging to practices within the lowest tertile have poorer outcomes. These findings suggest that guidelines aimed at reducing the use of gastroscopy may adversely affect cancer outcomes.

Competing interests None declared.

\section{OC-045 RANDOMISED PROSPECTIVE TRIAL OF TRANSNASAL VS STANDARD UPPER DIAGNOSTIC ENDOSCOPY UNDER LOCAL ANAESTHETIC: INTERIM ANALYSIS OF ENDOSCOPY QUALITY, PATIENT ACCEPTABILITY AND TOLERABILITY}

doi:10.1136/gutjnl-2012-302514a.45

E G Alexandridis, ${ }^{*}$ K Trimble, P Hayes, J N Plevris. Centre for Liver and Digestive Disorders, Royal Infirmary of Edinburgh, Edinburgh, UK

Introduction Transnasal upper gastrointestinal endoscopy (TNE) using ultrathin endoscopes is considered less invasive, thus an attractive alternative, if not a first choice option, for diagnostic upper endoscopy. This is the first prospective, randomised study, in a UK population to assess tolerability, acceptability and quality of TNE, in comparison with standard upper endoscopy (SOGD) under local anaesthetic.

Methods We prospectively recruited up to date 125 patients [59 male/66 female] mean age 57 years. The Fujinon EG530N (5.9 mm) and EG530WR $(9.4 \mathrm{~mm})$ endoscopes were used. The endoscopist and all patients completed detailed questionnaires regarding tolerability, acceptance and quality of each endoscopy using standard visual analogue scales (VAS). Oxygen saturation $\left[\mathrm{SaO}_{2}\right]$, heart rate [HR] and systolic blood pressure [SBP] were recorded during procedure. SOGD group received $\mathrm{O}_{2} 2 \mathrm{lt} / \mathrm{min}$. Quality of biopsies was evaluated blindly by the reporting pathologists.

Results Trial interim analysis included 129 endoscopies in 125 patients $[\mathrm{TNE}=65, \mathrm{SOGD}=64]$. In all patients intubation of $\mathrm{D} 2$ was achieved. VAS scores for patient comfort (higher score=greater comfort) were significantly better in the TNE compared to SOGD group ( 7 vs 5.6, respectively, $\mathrm{p}=0.0013$ ). 40 patients had previous experience of standard endoscopy, and $22(55 \%)$ reported gagging as main reason of discomfort. 22/40 were randomised to TNE. 21 of these 22 patients [95.5\%] stated preference to transnasal endoscopy in the future. Gagging score (higher score=more gagging) was significantly less in the TNE compared with SOGD group (0.05 vs 3.22 respectively, p2 (98\% vs $98.3 \%$, respectively, $\mathrm{p}=0.22$ ). Only 2 
(5.4\%) of TNE biopsies did not permit a definite diagnosis, but when standard biopsies were later taken, also failed to confirm diagnosis.

Conclusion TNE is equal to SOGD in image quality, endoscope handling and length of procedure. It is superior to SOGD in the ability to perform a panendoscopy and in terms of comfort and patient acceptance. Importantly it induced significantly less stress to the heart, thus should be considered as the endoscopic diagnostic method of choice in patients with significant cardio-respiratory problems.

Competing interests E Alexandridis conflict with: fellowship received from Fujinon, Imotech, K Trimble: None declared, P Hayes: None declared, J Plevris grant/research support from: Fujinon, Imotech, Conflict with: Fuji Provided Loan Equipment for trial.

\section{OC-046 GUIDELINES ON THE ENDOSCOPIC ASSESSMENT OF GASTRIC POLYPS: IS THE WORKLOAD JUSTIFIED?}

doi:10.1136/gutjnl-2012-302514a.46

T Jobson, ${ }^{*}$ P Thomas. Taunton and Somerset NHS FT, Taunton, UK

Introduction British Society of Gastroenterology guidelines on the management of gastric polyps ${ }^{1}$ recommend increased intensity of evaluation compared with traditional practice, including biopsy of ALL polyps, biopsy of intervening gastric mucosa (hyperplastic/ adenomatous polyps) and 1 year follow-up of dysplasia; a considerable increase in endoscopic and histological workload. The purpose is to reduce risk, and increase early detection of gastric carcinoma. It would therefore be expected that in clinical practice there would be an association between gastric polyp detection and subsequent carcinoma, and that application of the guidelines would improve patient outcomes. The purpose of this study was to evaluate whether this association exists in a DGH setting where traditional polyp assessment and surveillance was followed.

Methods Our database was searched for gastroscopies where polyps were found over 5 years. These records were cross-referenced against the local cancer database for those 5 years plus 1 year of follow-up. Any cases where polyps were found along with subsequent cancers were audited.

Results Details of 15489 gastroscopies in 11938 patients over 5 years (2006-2010) were analysed. 670 patients (756 gastroscopies) were found to have gastric polyps $(5.6 \%$-in line with larger studies). ${ }^{2}$ In 2006-2011, 1328 upper GI cancers were recorded on the local register. Cross reference of these revealed 57 patients with polyps at gastroscopy who had a co-existent record on the local cancer register. Cases were excluded where cancer was diagnosed at the index endoscopy [45 patients with polypoid tumours or coexistent polyps where the guidelines would not have influenced outcome]. Seven of the remainder had non-gastric neoplasia. Of the remaining 5,3 had a small neuroendocrine tumour on follow-up endoscopy, with no specific treatment. The other two were an 80 year old with a dysplastic polyp, followed up at 3 and 6 months when carcinoma was identified (the patient was not fit for radical treatment); and a patient with a large suspicious poly$\mathrm{p}$-inflammatory on initial biopsies with dysplasia identified following intensive follow-up (leading to resection). Neither would have benefited from application of the guidance.

Conclusion This retrospective analysis reveals no patients where an initial suspicion of simple fundic polyps was followed by a subsequent diagnosis of significant neoplasia, or where follow-up of hyperplastic polyps would prevent progression. On the basis of these results, the upper $95 \%$ confidence limit for patient benefit from the proposed algorithm is $\approx 0.5 \%$. These guidelines should therefore be more thoroughly assessed for cost effectiveness.

Competing interests None declared.

\section{REFERENCES}

1. Goddard AF, Badreldin R, Pritchard DM, et al. Gut 2010;59:1270-6.

2. Carmack SW, Genta RM, Schuler CM, et al. Am J Gastroenterol 2009;104:1524-32.

\section{OC-047 A PROSPECTIVE, BACK-TO-BACK, COMPARATIVE STUDY OF MANUAL SPIRAL ENTEROSCOPY AND DOUBLE- BALLOON ENTEROSCOPY}

doi:10.1136/gutjnl-2012-302514a.47

${ }^{1} \mathrm{E} J$ Despott, ${ }^{*} \mathrm{~A}$ Murino, ${ }^{1} \mathrm{~L}$ A Bourikas, ${ }^{1} \mathrm{M}$ Nakamura, ${ }^{2} \mathrm{~V}$ Ramachandra, ${ }^{1} \mathrm{C}$ Fraser. ${ }^{1}$ Wolfson Unit for Endoscopy, St Mark's Hospital and Academic Institute, Imperial College London, London, UK; ${ }^{2}$ Department of Anaesthesia and Intensive Care, NWLH NHS Trust, London, UK

Introduction By facilitating examination and therapy of the deep small bowel (SB) without recourse to surgery, double-balloon enteroscopy (DBE) has revolutionised SB endoscopy and is now considered to have become the "gold-standard" device assisted enteroscopy (DAE) technology. The more recently developed manual spiral enteroscopy (SE) shows promise to facilitate faster DAE, although whether these two technologies enable similar SB insertion depths remains uncertain.

Methods We performed a prospective, back-to-back comparative study of SE and DBE performed during the same session (under general anaesthesia) in 15 patients (10 women, 5 men; mean age $\pm \mathrm{SD}, 51.4 \pm 15.4$ years). The aim was to compare SB insertion depths by $\mathrm{SE}$ and $\mathrm{DBE}$ procedures performed in tandem, in the same cohort, using the same method of SB insertion depth estimation. Comparisons of duration, procedure difficulty (PD) (endoscopists' VAS score) and diagnostic yield (DY) were also performed. SE procedures were performed first and a tattoo was placed to mark the most distal point of SB insertion.

Results In 14 cases, the tattoo placed at SE was reached and passed by DBE; median (95\% CI) insertion depths for DBE vs SE were 265 (227 to 324 ) vs 175 (132 to 212 ) cm respectively, $p=0.004$. In one patient, the tattoo placed during SE could not be reached by DBE at an estimated final SB insertion depth of $160 \mathrm{~cm}$. Conversely, the median time taken to achieve deepest insertion was significantly shorter for SE as compared with DBE (median (95\% CI) times for SE vs DBE: 24 (20 to 28 ) min vs 45 (35 to 53 ) min respectively, $\mathrm{p}=0.0005$ ). Median procedure duration was also shorter for $\mathrm{SE}$ (median (95\% CI) duration: SE vs DBE was 28 (27 to 36 ) min vs 54 (45 to 62 ) $\mathrm{min}$ respectively, $\mathrm{p}=0.0002$. In the 14 patients where comparison was possible, median time taken by SE to reach maximal insertion was not significantly different to the time taken by $\mathrm{DBE}$ to reach the tattoo placed at SE (median $(95 \% \mathrm{CI}$ ) times for SE vs DBE were 24 (20 to 28) min vs 19 (14 to 26) min respectively, $\mathrm{p}=0.28$ ). Mean VAS PD scores were similar (mean $\mathrm{VAS} \pm \mathrm{SD}(95 \% \mathrm{CI}) \mathrm{PD}$ score: SE vs DBE, $5.3 \pm 2.3$ (4.0 to 6.6) vs $5.2 \pm 2.4$ (3.9 to 6.5) respectively, $\mathrm{p}=0.86$ ). Overall DY was similar for both (DY: SE vs DBE, $46.7 \%$ vs $53.3 \%$ respectively, $\mathrm{p}=0.4$ ). However, in one case, deeper SB insertion at DBE (median $\mathrm{SB}$ insertion at DBE vs $\mathrm{SE}, 270 \mathrm{~cm}$ vs $150 \mathrm{~cm}$ respectively) led to the identification of the suspect SB lesion (SB lipoma) which had not been reached by SE. Mild SE-related mucosal trauma was observed in $60 \%$ of cases; no other complications were observed in the study.

Conclusion Our experience suggests that although SE may enable faster DAE procedure times, DBE allows for significantly deeper SB insertion.

Competing interests E Despott grant/research support from: Fujifilm \& Imotech Medical (UK), A Murino grant/research support from: Fujifilm \& Imotech Medical (UK), L Bourikas: None declared, M Nakamura: None declared, V Ramachandra: None declared, C Fraser: None declared. 